\title{
PRONTUÁRIO ONCOLÓGICO: INSTRUMENTO DE INFORMAÇÃO DA DOENÇA E DO TRATAMENTO
}

\author{
MEDICAL RECORD IN ONCOLOGY: AN INSTRUMENT \\ FOR DISEASE AND TREATMENT INFORMATION
}

\author{
Bianca Pozza dos Santos ${ }^{\mathrm{a}^{*}}$, Eda Schwartz ${ }^{\mathrm{b}^{*}}$, Aline Machado Feijo ${ }^{\mathrm{c}^{*}}$, Rosani Manfrin Muniz ${ }^{\mathrm{d}^{*}}$, \\ Juliana Graciela Vestena Zillmer ${ }^{\mathrm{e}^{*}}$, Aline da Costa Viegas ${ }^{\mathrm{f}^{*}}$ \\ abi.santos@bol.com.br, beschwartz@terra.com.br, caline_feijo@yahoo.com.br, dromaniz@terra.com.br, \\ ejuzillmer@gmail.com, falinecviegas@hotmail.com \\ *Faculdade de Enfermagem da Universidade Federal de Pelotas - Pelotas (RS), Brasil
}

Data de recebimento do artigo: 02/02/2015

Data de aceite do artigo: 06/04/2015

\section{RESUMO}

Introdução: Conhecer a epidemiologia do câncer é essencial para a realização de açôes, e um dos meios para o conhecimento é a consulta ao prontuário. Assim, há a necessidade de avaliar a qualidade das informações disponíveis nos prontuários de pacientes oncológicos, os quais possibilitarão a continuidade da assistência. Objetivo: Descrever os dados contidos no prontuário de um serviço de oncologia, segundo características demográficas, clínicas e do tratamento quimioterápico. Materiais e métodos: Estudo quantitativo realizado em um serviço de oncologia. Os participantes que estavam em tratamento quimioterápico responderam o instrumento com 82 questôes sociodemográficas. Os dados sobre a doença e o tratamento foram coletados no prontuário. Para a análise, realizou-se a codificação das variáveis, elaborou-se um banco de dados e o controle de qualidade ocorreu por dupla digitação. Resultados: A amostra incluiu 221 prontuários, sendo analisados 117 prontuários de pessoas do sexo feminino e 104 do masculino. A faixa etária predominante foi entre 51 e 60 anos. A incidência de câncer de mama nas mulheres foi de $41 \%$, e de intestino nos homens, 23\%, prevalecendo o estadiamento IV. Foi evidenciada a finalidade quimioterápica paliativa para o tratamento da doença. Em relação ao período de planejamento do tratamento quimioterápico, prevaleceu o de um a doze meses, e quanto aos ciclos quimioterápicos, foi o primeiro. Conclusóes: Muitos dados estavam incompletos nos prontuários, levando à carência de informaçóes, o que pode dificultar as açôes de cuidado.

Palavras-chave: Oncologia; quimioterapia; registros médicos; enfermagem.

\section{ABSTRACT}

Introduction: Knowing the epidemiology of cancer is essential to perform actions, and one of the ways to do this is consulting medical records. As a result, it is necessary to evaluate the quality of available information in the oncological patients' medical records, which will enable the continuity of assistance. Objective: To describe the data in the medical records from an oncological service, according to demographic and clinical characteristics, and to chemotherapy. Materials and methods: It is a quantitative study performed at an oncological service. The participants under chemotherapy answered 82 sociodemographic questions. The data about the disease and the treatment was collected from the medical records. For the analysis, we performed a variable of codifications, elaborated a database, and the quality control occurred throughout double typing. Results: The sample had 221 medical records, from which 117 were from women, and 104 from men. The predominant age group was between 51 and 60 years old. The incidence of breast cancer in women was $41 \%$, and colon cancer in men, $23,0 \%$, with the prevalence of stage IV. It was highlighted the palliative chemotherapy for the treatment of the disease. About the planning period of chemotherapy, one to twelve months preponderated, and the chemotherapy cycles were the first. Conclusions: Several data were incomplete in the medical records, which takes us to lack of information, and can complicate care actions.

Keywords: Medical oncology; drug therapy; medical records; nursing. 


\section{Introdução}

Câncer é uma palavra latina e advém de "cancri", que significa "caranguejo". A simbologia desse animal relacionada a tal doença remete ao fato de suas patas serem comparadas à característica infiltrativa ${ }^{1}$. Para a Organização Mundial da Saúde (OMS), o câncer é um processo de disseminação incontrolável de células, podendo surgir em qualquer lugar do corpo ${ }^{2}$.

Segundo o Instituto Nacional de Câncer (INCA), ele abarca mais de 100 doenças que se caracterizam pelo crescimento celular desordenado, pela multiplicação rápida, e consequentemente, pela invasão nos tecidos e nos órgãos. Nesse contexto, é importante destacar a existência de muitos fatores que geram o câncer no organismo, podendo ser externos (meio ambiente e hábitos de vida) e/ou internos (genética), porém ambos podem se inter-relacionar ${ }^{3}$.

No Brasil, desde o final do século XX, o câncer tem se destacado como uma das principais causas de morbimortalidade ${ }^{4}$. Já em nível mundial, mais de $11 \mathrm{mi}-$ lhóes de pessoas são diagnosticadas todos os anos². Em virtude desse elevado número, o Ministério da Saúde lançou, em 8 de dezembro de 2005, a Portaria GM/MS no 2.439, condizente à Política Nacional de Atenção Oncológica, com a finalidade de garantir o atendimento integral a qualquer pessoa com câncer, por meio das unidades ou dos centros de assistência em oncologia, presentes no Brasil, envolvendo profissionais especializados e capacitados para determinar a extensão da doença, tratar, cuidar e assegurar a qualidade dos serviços de assistência oncológica ${ }^{5}$.

Conhecer a epidemiologia do câncer é essencial para a realização de açôes que visem à promoção da saúde, à prevenção, à detecção precoce da doença, além de definir metas e de avaliar resultados de atençáo oncológica em todos os níveis de saúde ${ }^{6-7}$. Assim, um dos meios para o conhecimento desses dados é a consulta ao prontuário, o que permite que o profissional da saúde concentre sua assistência na prevenção de possíveis agravos que podem ocorrer com a evolução da doença, e intervir precocemente para minimizar prováveis complicaçôes decorrentes da terapêutica estabelecida.

Além do mais, um sistema de informação adequado permite o acompanhamento do percurso de atendimento do paciente em um determinado cenário e também a possibilidade de mobilização e de integração dos dados da pessoa, objetivando descrever e modelar essa trajetória de cuidado ${ }^{8}$.

O prontuário consiste em um veículo essencial para a comunicação entre os membros da equipe multiprofissional ${ }^{9}$, permitindo o acompanhamento da saúde do paciente e garantindo a continuidade do cuidado durante 24 horas. $\mathrm{O}$ ato de registrar trata-se de um resgate do trabalho profissional acerca da assistência prestada. Desse modo, os registros se tornam fundamentais para um acompanhamento do tratamento e do atendimento ao paciente oncológico. Assim, é importante o enfermeiro gerenciá-los nos prontuários, além de ter conhecimento científico sobre a progressão da doença e a finalidade do tratamento estabelecido, de modo a promover qualidade nas açóes de cuidado ${ }^{10}$.

No entanto, salienta-se que uma parcela das informações referentes à dimensão da doença, dos resultados dos métodos preventivos, do rastreamento precoce, do tratamento e dos cuidados, encontra-se fragmentada em alguns sistemas ou registros de dados ${ }^{11}$. Quando as anotaçôes são escassas ou inadequadas, podem comprometer a assistência prestada ao paciente, bem como à equipe profissional e à instituição ${ }^{12}$.

A qualidade dos registros sobre o atendimento aos pacientes é uma preocupação crescente nas instituições. As informaçóes insuficientes afetam e interpóem-se à qualidade do cuidado realizado ${ }^{13}$. Portanto, entende-se que as características da doença e da terapêutica estabelecida devem estar devidamente registradas.

Entretanto, em uma pesquisa ${ }^{14}$ foi descrito que há poucos estudos avaliando a qualidade das informações disponíveis nos prontuários, fato associado às conhecidas barreiras existentes ao se coletar dados desses documentos para o acompanhamento dos pacientes, levando-se em conta a ausência de dados ou sua inconformidade. Dessa maneira, pontuam que o conhecimento da fidedignidade e a completude dos prontuários permitem uma melhor utilização das informações, podendo qualificar as pesquisas.

Nesse sentido, o objetivo deste estudo consiste em descrever os dados contidos no prontuário de um serviço de oncologia, segundo características demográficas, clínicas e do tratamento quimioterápico.

\section{Metodologia}

Este estudo apresenta uma abordagem quantitativa a partir do trabalho monográfico Análise de prontuários de um serviço de oncologia: caracterização dos pacientes com câncer em tratamento quimioterápico, sendo um recorte da pesquisa intitulada "Os clientes oncológicos e suas famílias e os sistemas de cuidado nas condiçóes crônicas".

A coleta de dados ocorreu entre março e junho de 2010 no Serviço de Oncologia do Hospital Escola da Universidade Federal de Pelotas (HE/UFPel). Constituíram a amostra do estudo, por conveniência, 221 pacientes oncológicos que atendiam aos seguintes critérios de inclusão: ter idade igual ou superior 
a 18 anos; estar ciente do diagnóstico de câncer e do tratamento.

Primeiramente, os participantes responderam o instrumento com 82 questóes sociodemográficas, que foi aplicado para todos os pacientes que estavam em tratamento no período. Posteriormente, os dados sobre a doença oncológica e o tratamento quimioterápico foram coletados no prontuário. A coleta de dados foi realizada por acadêmicas de enfermagem previamente capacitadas.

O controle de qualidade dos dados ocorreu em todo o período da coleta, por meio da revisão de cada instrumento pelos supervisores no momento da entrega dos questionários, para detectar e corrigir possíveis erros e contradições nas respostas registradas. A reaplicação do questionário reduzido foi efetivada por meio de contatos telefônicos a $10 \%$ dos participantes.

Para a análise dos dados, realizou-se a codificação apropriada de cada uma das variáveis contidas no instrumento quantitativo. Elaborou-se um banco de dados no programa Epi Info versão 6.04, e o controle de qualidade ocorreu por intermédio de dupla digitação, a fim de detectar possíveis inconsistências. Logo, os resultados foram transferidos para o programa Stata 9.1, por meio do Stata Transfer. Para a análise estatística, foi realizada a distribuição da frequência das variáveis independentes utilizadas para este estudo, tais como: sexo, idade, câncer, estadiamento, finalidade quimioterápica, planejamento e ciclo quimioterápico.

Seguiram-se os princípios da Resolução 196/96 do Conselho Nacional de Saúde do Ministério da Saúde, referente às diretrizes sobre pesquisa com seres humanos ${ }^{15}$. Aos participantes do estudo foram apresentados os objetivos da pesquisa, por meio do Termo de Consentimento Livre e Esclarecido, assegurando-lhes o anonimato, o livre acesso aos dados e aos resultados da pesquisa, e o direito de desistir de participar em qualquer momento. O projeto foi aprovado pelo Comitê de Ética em Pesquisa da Universidade Católica de Pelotas sob o número 2008/23 e registrado no COCEPE sob o número 4.04.01.015.

\section{Resultados}

Dos 221 prontuários dos pacientes oncológicos analisados que estavam em tratamento quimioterápico no serviço de oncologia, 117 pertenciam ao sexo feminino e 104 ao sexo masculino.

Com relação à idade, a faixa etária predominante nas mulheres situou-se entre 51 e 60 anos, com média de 55,6 anos, representando 33,3\% da amostra. Para os homens, a faixa etária foi de 61 a 70 anos, com média de 61,2 anos, $27,8 \%$.
Sobre a localização do sítio primário do câncer, nas mulheres se destacou o de mama com $41,0 \%$, seguido pelo intestino $(18,8 \%)$, pulmão $(12,8 \%)$ e colo do útero $(8,5 \%)$. Já nos homens, a doença situou-se em primeiro lugar no intestino $23,0 \%$, seguido pela próstata $(18,2 \%)$, pulmáo $(17,3 \%)$ e sangue $(15,3 \%)$, estando demonstrado na Tabela 1.

Tabela 1: Distribuição da faixa etária e da localização do sítio primário do câncer nos sexos feminino e masculino

\begin{tabular}{|c|c|c|c|c|}
\hline & \multicolumn{2}{|c|}{ Feminino $(N=117)$} & \multicolumn{2}{|c|}{$\begin{array}{c}\text { Masculino } \\
(\mathrm{N}=104)\end{array}$} \\
\hline & $\mathbf{N}$ & $(\%)$ & $\mathbf{N}$ & $(\%)$ \\
\hline \multicolumn{5}{|l|}{ Faixa Etária } \\
\hline 20 a 30 anos & 3 & 2,5 & 2 & 1,9 \\
\hline 31 a 40 anos & 13 & 11,1 & 4 & 3,8 \\
\hline 41 a 50 anos & 21 & 17,9 & 16 & 15,3 \\
\hline 51 a 60 anos & 39 & 33,3 & 24 & 23,0 \\
\hline 61 a 70 anos & 26 & 22,2 & 29 & 27,8 \\
\hline 71 a 80 anos & 13 & 11,1 & 24 & 23,0 \\
\hline Acima de 81 anos & 2 & 1,7 & 5 & 4,8 \\
\hline \multicolumn{5}{|l|}{$\begin{array}{l}\text { Sítio Primário do } \\
\text { Câncer }\end{array}$} \\
\hline Pele & 2 & 1,9 & 2 & 1,7 \\
\hline Mama & 48 & 41,0 & 1 & 0,9 \\
\hline Próstata & 0 & 0,0 & 19 & 18,2 \\
\hline Pulmão & 15 & 12,8 & 18 & 17,3 \\
\hline Intestino & 22 & 18,8 & 24 & 23,0 \\
\hline Colo do útero & 10 & 8,5 & 0 & 0,0 \\
\hline Estômago & 1 & 0,8 & 3 & 2,8 \\
\hline Esôfago & 1 & 0,8 & 6 & 5,7 \\
\hline Ovário & 5 & 4,2 & 0 & 0,0 \\
\hline Fígado/Vesícula & 0 & 0,0 & 1 & 0,9 \\
\hline Rins & 2 & 1,7 & 0 & 0,0 \\
\hline Sangue/Linfoma & 5 & 4,2 & 16 & 15,3 \\
\hline Cavidade oral & 1 & 0,8 & 3 & 2,8 \\
\hline Cérebro & 0 & 0,0 & 1 & 0,9 \\
\hline Bexiga & 1 & 0,8 & 3 & 2,8 \\
\hline Testículo & 0 & 0,0 & 2 & 1,9 \\
\hline Retroperitônio & 1 & 0,8 & 0 & 0,0 \\
\hline Sítio desconhecido & 3 & 2,5 & 5 & 4,8 \\
\hline
\end{tabular}

Quanto ao estadiamento do câncer, observou-se que, dos 221 prontuários investigados, 213 continham essa informação, predominando o estádio IV em ambos os gêneros, $35,4 \%$ nas 113 mulheres, e 50,0\% nos 100 homens.

Além disso, a informação sobre a finalidade quimioterápica recebida pelos pacientes foi encontrada em 194 prontuários, dos quais 106 eram de mulheres que realizavam a modalidade paliativa $(42,9 \%)$ e a adjuvante $(42,0 \%)$. Desencadeando o mesmo nos 88 homens, com $56,7 \%$ na modalidade paliativa e $19,5 \%$ na adjuvante. 
Em relação ao período de planejamento do tratamento quimioterápico do paciente, apenas 170 prontuários analisados tinham o tempo de seguimento, sendo de 1 a 12 meses para as 88 mulheres, representado por $92,6 \%$, e para os 69 homens, $92,0 \%$.

Já os ciclos quimioterápicos foram encontrados em 171 prontuários. Inclusive, observou-se que, além da ausência desse dado em alguns documentos, não havia o registro do período específico que compreendia o ciclo, ou seja, se era constituído por dias, semanas ou meses. Nesse caso, em ambos os sexos, a maior frequência encontrava-se nos que estavam no primeiro ciclo de tratamento quimioterápico, representado por $47,3 \%$ das 45 mulheres, e $56,5 \%$ dos 43 homens.

Tabela 2: Frequência do estadiamento do câncer, do tipo de quimioterapia, do planejamento quimioterápico e dos ciclos em ambos os sexos.

\begin{tabular}{|c|c|c|c|c|}
\hline \multirow{2}{*}{ Estadiamento } & \multicolumn{2}{|c|}{ Feminino $(\mathrm{N}=113)$} & \multicolumn{2}{|c|}{ Masculino $(\mathrm{N}=100)$} \\
\hline & $\mathbf{N}$ & $(\%)$ & $\mathbf{N}$ & $(\%)$ \\
\hline I & 6 & 5,3 & 3 & 3,0 \\
\hline II & 38 & 33,6 & 13 & 13,0 \\
\hline III & 29 & 25,6 & 34 & 34,0 \\
\hline IV & 40 & 35,4 & 50 & 50,0 \\
\hline Tipo de & \multicolumn{2}{|c|}{ Feminino $(\mathrm{N}=106)$} & \multicolumn{2}{|c|}{ Masculino (N=88) } \\
\hline Quimioterapia & $\mathrm{N}$ & $(\%)$ & $\mathrm{N}$ & $(\%)$ \\
\hline Neoadjuvante & 14 & 13,0 & 9 & 9,2 \\
\hline Adjuvante & 45 & 42,0 & 19 & 19,5 \\
\hline Paliativa & 46 & 42,9 & 55 & 56,7 \\
\hline Curativa & 1 & 1,8 & 5 & 14,4 \\
\hline Planejamento & \multicolumn{2}{|c|}{ Feminino $(\mathrm{N}=95)$} & \multicolumn{2}{|c|}{ Masculino (N=75) } \\
\hline quimioterápico & $\mathbf{N}$ & (\%) & $\mathbf{N}$ & $(\%)$ \\
\hline 1 a 12 meses & 88 & 92,6 & 69 & 92,0 \\
\hline 13 a 24 meses & 5 & 5,2 & 3 & 4,0 \\
\hline 25 a 36 meses & 2 & 2,1 & 2 & 2,6 \\
\hline 37 a 48 meses & 0 & 0,0 & 1 & 1,3 \\
\hline Ciclos & \multicolumn{2}{|c|}{ Feminino $(\mathrm{N}=95)$} & \multicolumn{2}{|c|}{ Masculino $(\mathrm{N}=76)$} \\
\hline quimioterápicos & $\mathrm{N}$ & $\%$ & $\mathrm{~N}$ & $\%$ \\
\hline Primeiro & 45 & 47,3 & 43 & 56,5 \\
\hline Segundo & 14 & 14,7 & 18 & 23,6 \\
\hline Terceiro & 8 & 8,4 & 5 & 6,5 \\
\hline Quarto & 6 & 6,3 & 3 & 3,9 \\
\hline Quinto & 7 & 7,3 & 2 & 2,6 \\
\hline Sexto & 7 & 7,3 & 2 & 2,6 \\
\hline Sétimo em diante & 8 & 8,4 & 3 & 3,9 \\
\hline
\end{tabular}

\section{Discussão}

Para a concretização deste estudo, adotou-se como foco principal a procura pelos dados informativos sobre a doença oncológica a partir dos prontuários dos pacientes em tratamento quimioterápico, em um serviço de alta complexidade em oncologia. A amostra total foi de 221 prontuários impressos e/ou eletrônicos, mas nem todos continham os dados que eram procurados, tais como o estadiamento, o tipo de quimioterapia, o planejamento e os ciclos quimioterápicos. Todavia, constatou-se em todos os documentos que a faixa etária mais incidente de câncer estabeleceu-se entre a quinta e a sétima década de vida.

Apesar dos progressos da medicina em relação à cura de várias doenças infectocontagiosas, aumentando a expectativa de vida da população, as chances de ter uma enfermidade crônica também aumentaram. Como é o caso do câncer, definido como uma doença crônica não transmissível que necessita de um período para o seu desenvolvimento, o que faz elevar a sua incidência com o avançar dos anos ${ }^{10}$.

Ressalta-se que a oncologia e a idade estão inter-relacionadas. A incidência de câncer aumenta proporcionalmente de acordo com a longevidade; assim, quanto mais a população envelhece, a tendência no crescimento do número de casos novos de câncer se concretiza ${ }^{16}-$ fato constatado em um estudo transversal e descritivo, apontando que a idade média dos pacientes oncológicos foi de 56,6 anos, estando a faixa etária concentrada acima dos 60 anos $^{17}$.

Sobre o tipo de câncer incidente neste estudo, notou-se que na população feminina o câncer de mama é o mais presente, seguido pelo câncer de intestino. Nos homens, destacaram-se o de intestino e o de próstata. De acordo com o Instituto Nacional de Câncer (INCA), os tipos mais incidentes de câncer, exceto o de pele não melanoma, são os de mama feminina, seguido de próstata, cólon e reto, pulmão, estômago e colo do útero ${ }^{18}$.

Corroborando um estudo que descreveu o perfil clínico dos pacientes em tratamento quimioterápico ambulatorial, o principal tipo de câncer apresentado foi o de mama, acometendo 35,6\% das mulheres, seguido do câncer de cólon e reto, com $20,7 \%$, próstata $(17,3 \%)$, cânceres hematológicos $(6,9 \%)$, pulmão $(4,6 \%)$, esôfago $(3,4 \%)$ e outros diversos com menor representatividade estatística, totalizando $11,5 \%{ }^{19}$.

Enfatiza-se que os tipos de câncer mais frequentes neste e em outros estudos são aqueles que têm campanhas educativas visando a sua prevenção, sendo muito comuns no dia a dia das pessoas. Todavia, representam uma das maiores preocupaçôes mundiais de saúde pública, em virtude de sua alta incidência ${ }^{20}$.

Quanto ao estadiamento do câncer, destacou-se em ambos os sexos o estádio IV, levando os pacientes a realizarem a quimioterapia com finalidade paliativa. Sabe-se que a quimioterapia é um tipo de tratamento sistêmico para o câncer, que tem a função de evitar a proliferação 
de células cancerígenas, impedindo a disseminação no organismo ${ }^{21}$. O seu tempo de aplicação dependerá do que o oncologista determinar, destacando-se que o período de seguimento encontrado foi de 1 a 12 meses, estando a maioria dos pacientes no primeiro ciclo.

Como neste estudo predominou a finalidade paliativa, destaca-se que tem como objetivo diminuir ou amenizar a severidade dos sintomas causados pela doença, sem remover a sua origem e tampouco efetuar uma cura, mas manter o controle, no intuito de promover a longevidade e o bem-estar ${ }^{22}$. A adoção do tratamento quimioterápico paliativo compreende um dado significante que está relacionado às problemáticas que envolvem as ações de prevenção primária e secundária da doença oncológica, interferindo diretamente no tempo de sobrevida e na qualidade de vida dos pacientes ${ }^{19}$.

Ainda observou-se que o estádio IV é o predominante. Assim, definir o estadiamento do câncer é um dos princípios fundamentais para a adoção do tratamento. Entretanto, atrasos que retardam o diagnóstico e/ou o estabelecimento da terapêutica levam ao crescimento tumoral, com possível prejuízo às chances de cura dos pacientes $^{23}$. Isso faz que se passe a adotar medidas, para minimizar a sintomatologia da doença, que promovam apenas a qualidade de vida ${ }^{10}$.

Dados semelhantes aos deste estudo também foram encontrados em uma pesquisa na qual o tratamento paliativo foi o mais utilizado (36,9\%), seguido do adjuvante $(28,7 \%)$. Com relação à fase da doença oncológica, em 11,5\% dos prontuários não foram encontrados registros sobre o estadiamento. Entretanto, ao se considerar a classificação TNM, sendo T relativo ao tumor, $\mathrm{N}$ ao comprometimento de linfonodos e $\mathrm{M}$ à presença de metástases, constatou-se que $25,3 \%$ estavam no estádio avançado, isto é, estádio IV ${ }^{19}$.

Estabelecer um estadiamento correto é de extrema relevância para o prognóstico e para determinar a melhor conduta terapêutica para a doença $\mathrm{a}^{24}$. Nesse contexto, a quimioterapia compóe uma das formas de tratamento mais escolhidas, que almeja a cura, o controle e a paliação $0^{25}$, podendo ser combinada com a cirurgia e a radioterapia ${ }^{26}$. Salienta-se ainda, que para a instituição da quimioterapia, adotam-se períodos denominados de ciclos, os quais são designados ao paciente com câncer no intuito de atuar no controle ou na prevenção de novas células cancerígenas ${ }^{10}$.

Como neste estudo muitos dados sobre a doença e o tratamento não foram encontrados nos prontuários, é importante destacar a importância de uma melhor documentação das açóes e das intervençóes realizadas, salientando que os registros devem traduzir o máximo de conhecimento sobre as condiçôes de saúde dos pacientes, incluindo-se os aspectos referentes aos procedimentos, às necessidades, às queixas e à evolução do estado de saúde-doença. As anotaçóes comprovam a assistência prestada ${ }^{13}$, não sendo somente função do enfermeiro liderar e acompanhar a efetivação dos registros, mas, sim, de todos os profissionais que prestam assistência.

Quando não há informaçóes referentes ao tratamento oncológico e à conduta prestada, a probabilidade do agravamento da doença passa a ser elevada. Assim, cada açáo efetuada ao paciente deve ser minuciosamente registrada nos prontuários, para que o cuidado possa ser executado de forma ágil e objetivo, dando continuidade à assistência e ao entendimento do processo saúde-doença. Ademais, cada anotação feita deverá estar legível e conter a identificação do profissional responsável pelo atendimento, acompanhado da assinatura e o número do conselho representativo.

Nesse contexto, a preocupação em construir este estudo a partir dos dados coletados no prontuário ocorreu pela conscientização de que esse documento agrega as açóes desempenhadas pelos profissionais de saúde, no que diz respeito à história da doença e ao planejamento do tratamento. No entanto, como foi observado, muitas vezes esses documentos, tanto os impressos quanto os eletrônicos, estão carentes de informaçóes em sua completude, impossibilitando a melhor assistência à saúde para o paciente oncológico.

Corroborando os achados desta pesquisa, um estudo descritivo reuniu 105 instituiçóes hospitalares brasileiras de ensino, filiadas à Associação Brasileira de Hospitais Universitários e a Entidades de Ensino (ABRAHUE), que tinha por finalidade analisar 77 prontuários recebidos no início de novembro de 2003 até o final de agosto de 2004. A maioria dos prontuários apresentava deficiência de informação com relação à identificação do paciente, anamnese, exame físico, diagnóstico médico, conduta terapêutica, entre outros. Esse fato trouxe preocupação, pois os documentos eram provenientes de instituiçōes universitárias (assim como neste estudo), que ocupam o ápice da atenção à saúde, sendo representadas pelo nível terciário, o que nos leva a refletir sobre o que possivelmente seria encontrado nos prontuários dos serviços de saúde de atenção primária e secundária no Brasil ${ }^{27}$.

A falta de informaçóes registradas sobre o paciente, principalmente em relação ao histórico do processo saúde-doença, dificulta a ação de profissionais que necessitam ter conhecimentos fidedignos sobre a evolução da doença e a resposta ao tratamento instituído. Conhecer os dados informativos sobre a incidência da doença é essencial para determinar prioridades em programas estratégicos de prevenção e de tratamento, auxiliar os gestores de saúde no direcionamento de investimento e adoção de recursos, além de definir condutas e analisar os resultados encontrados ${ }^{7}$. 
Além de todas as questôes legais, registros precários dificultam a continuidade e a avaliação da assistência. Assim é fundamental que não só o enfermeiro passe por orientação e capacitação, mas todos os profissionais de saúde, para que compreendam a importância dos registros e colaborem para que sejam elaborados de forma efetiva ${ }^{13}$. Todavia, pode haver dificuldades para a educação da equipe multiprofissional quanto à importância em registrar dados, o que talvez seja justificado pela sobrecarga de trabalho.

Nesse sentido, um estudo desenvolvido na França aponta que a utilização de sistemas de informação permite o conhecimento da trajetória de cuidado, além de possibilitar o cruzamento com dados clínicos, estabelecendo uma avaliação do cuidado prestado. Também pode haver produção de indicadores que contribuem para melhorar significativamente o planejamento do cuidado, e garantir que todos os pacientes tenham acesso ao tratamento adequado ${ }^{8}$.

\section{Conclusão}

Entre os dados encontrados neste estudo, revelou-se a presença real do câncer na fase adulta avançada, em que os tipos incidentes de neoplasias foram aqueles que possuem campanhas educativas de prevençáo, como o de mama, de colo de útero, de próstata, entre outros. Outro fator preocupante é que a maioria dos pacientes em tratamento quimioterápico estava com a doença em estádio avançado, tornando muitas vezes difícil a busca pela cura. Nesse ínterim, passam a depender de tratamentos que podem perdurar por semanas, meses ou anos, visando à amenização da sintomatologia gerada pelo câncer, como os que possuem finalidade paliativa.

Apesar dos achados, é importante ressaltar que algumas limitaçôes do estudo se devem à falta de informações presentes nos prontuários. Dessa forma, a carência de dados pode dificultar as açóes de cuidado. Sendo assim, salienta-se a importância de uma atenção mais eficaz no preenchimento completo dos prontuários, de modo a auxiliar uma melhor compreensão do estado de saúde do paciente, de possíveis estratégias terapêuticas a serem adotadas e/ou de programas que tenham em vista o comprometimento de favorecer a qualidade na assistência.

O prontuário é um documento que abrange todo $\mathrm{o}$ ato assistencial, promovendo uma atenção integral e garantindo a continuidade da ação. Desse modo, é imprescindível mantê-lo corretamente preenchido, pois é um precioso instrumento auxiliar para a investigação, e, para fins éticos, comprova a presteza e o atendimento estabelecido. Relaciona-se também com a continuidade da assistência, facilidade de fazer o diagnóstico correto, de avaliar mais rapidamente o paciente, com a segurança de saber quais foram as condutas já tomadas e as que precisam de continuidade.

\section{Referências}

1. Stumm EMF, Leite MT, Maschio G. Vivências de uma equipe de enfermagem no cuidado a pacientes com câncer. Cogitare enferm. 2008;13(1):75-82.

2. World Health Organization [internet]. [acesso em: 12 set 2010]. Disponível em: <http://www.who.int/features/ qa/15/es/index.html>.

3. Brasil MS. Estimativa 2012: Incidência de Câncer no Brasil. Rio de Janeiro: INCA; 2011.

4. Silva JFS, Mattos IE. Padrão de distribuição do câncer em cidade da zona de fronteira: tendência da mortalidade por câncer em Corumbá, Mato Grosso do Sul, no período 1980-2006. Epidemiol serv. saude. 2011;20(1):65-74.

5. Brasil [internet]. Brasília: Ministério da Saúde (BR). Portaria GM/MS no 2.439. [acesso em: 20 set 2010]. Disponível em: <http://dtr2001.saude.gov.br/sas/PORTARIAS/Port2005/ GM/GM-2439.htm>.

6. Tonani M, Carvalho EC. Risco de câncer e comportamentos preventivos: a persuasão como estratégia de intervenção. Rev latino-am enfermagem. 2008;16(5):864-70.

7. Fonseca AJ, Ferreira LP, Dalla-Benetta AC, Roldan CN, Ferreira MLS. Epidemiologia e impacto econômico do câncer de colo de útero no Estado de Roraima: a perspectiva do SUS. Rev bras Ginecol Obstet. 2010;32(8):386-92.

8. Defossez G, Rollet A, Dameron O, Ingrand P. Temporal representation of care trajectories of cancer patients using data from a regional information system: an application in breast cancer. BMC Med Inform and Decis Making. 2014;14:24.

9. Massad E, Marin HF, Azevedo Neto RS. O prontuário eletrônico do paciente na assistência, informação e conhecimento médico. São Paulo: H. de F. Marin; 2003.

10. Santos BP. Análise de prontuários de um Serviço de Oncologia: caracterização dos pacientes com câncer em tratamento quimioterápico [monografia]. Pelotas: Faculdade de Enfermagem/UFPel; 2011.

11. Souza RC, Freire SM, Almeida RT. Sistema de informação para integrar os dados da assistência oncológica ambulatorial do Sistema Único de Saúde. Cad. saúde publica. 2010;26(6):1131-40.

12. Setz VG, Dílnnocenzo M. Avaliação da qualidade dos registros de enfermagem no prontuário por meio da auditoria. Acta paul enferm. 2009;22(3):313-7.

13. Silva JA, Grossi ACM, Haddad MCL, Marcon SS. Avaliação da qualidade das anotaçôes de enfermagem em unidade semiintensiva. Esc Anna Nery. 2012;16(3):576-81.

14. Sarmento RMB, Obadia RCM, Camacho PG, Rocha MRL, Thuler LCS. Fidedignidade e completude dos prontuários médicos em relação aos eventos ou reaçôes adversas 
em pesquisa clínica. Rev bras cancerol. 2011;57(4):535-40.

15. Brasil MS. Conselho Nacional de saúde. Diretrizes e normas regulamentadoras de pesquisa envolvendo seres humanos. Resolução n. ${ }^{\circ}$ 196, de 10 de outubro de 1996. Brasília; 1996.

16. Visentin A, Lenardt MH. O itinerário terapêutico: história oral de idosos com câncer. Acta paul enferm. 2010;23(4):486-92.

17. Azevedo CD, Dal Bosco SM. Perfil nutricional, dietético e qualidade de vida de pacientes em tratamento quimioterápico. ConScientiae Saúde. [Internet] 2011. [acesso em: 10 jul 2014]. Disponível em: <http://www.redalyc.org/articulo. oa?id=92917188004>.

18. Brasil MS. Instituto Nacional de Câncer José Alencar Gomes da Silva (Brasil). Estimativa 2014 - Incidência de Câncer no Brasil. Rio de Janeiro: INCA; 2014.

19. Silva MM, Silva JA, Esteves LO, Mesquita MGR, Stipp MAC, Duarte SCM. Perfil sociodemográfico e clínico de pessoas em tratamento quimioterápico: subsídios para o gerenciamento em enfermagem. Rev. eletr enf [Internet]. 2013 jul/set. 15(3):704-12. [acesso em: 12 jun 2014]. Disponível em: <http://dx.doi.org/10.5216/ree.v15i3.18417>.

20. Costa MCC, Teixeira LA. As campanhas educativas contra o câncer. Hist cienc saúde -manguinhos. 2010;17(1):223-41.
21. Fernandes IC, Mello AA. Entendendo e combatendo o câncer. Tema. 2008;7(10/11):02-11.

22. Singh A. Medicina moderna: rumo à prevenção, à cura, ao bem-estar e à longevidade. Rev latinoam psicopatol fundam. 2010;13(2):265-82.

23. Trufelli DC, Miranda VC, Santos MBB, Fraile NMP, Pecoroni PG, Gonzaga SFR, et al. Análise do atraso no diagnóstico e tratamento do câncer de mama em um hospital público. Rev assoc med bras. 2008;54(1):72-6.

24. Murta EFC, Rosekeila S. Métodos de imagem no estadiamento das neoplasias malignas de colo uterino. Femina. 2010;38(1):41-6.

25. Sawada NO, Nicolussi AC, Okino L, Cardozo FMC, Zago MMF. Avaliação da qualidade de vida de pacientes com câncer submetidos à quimioterapia. Rev esc enferm USP. 2009;43(3):581-7.

26. Brasil [internet]. Brasília: Ministério da Saúde (BR). Quimioterapia. [acesso em: 12 out 2010]. Disponível em: <http://www.inca.gov.br/conteudo_view. asp? ID=101\#topo $>$.

27. Silva FG, Tavares-Neto J. Avaliação dos prontuários médicos de hospitais de ensino do Brasil. Rev bras educ med. 2007;31(2):113-26.

\section{Como citar este artigo:}

Santos BP, Schwartz E, Feijó AM, Muniz RM, Zillmer JCV, Viegas AC. Prontuário oncológico: instrumento de informação da doença e do tratamento medical. Rev. Aten. Saúde. 2015;13(46):99-105 